\title{
IDENTIFICACIÓN FACIAL DE EMOCIONES: UTILIDAD EN LOS CUERPOS Y FUERZAS DE SEGURIDAD (POLICÍAS LOCALES)
}

\author{
FACIAL IDENTIFICATION OF EMOTIONS: UTILITY IN BODIES AND \\ SECURITY FORCES (LOCAL POLICE)
}

\author{
Juan Ángel Antal \\ Licenciado en Criminología \\ Experto en Comunicación Verbo-Corporal y Detección de Engaño \\ juanangelanta@gmail.com
}

\section{SUMARIO}

- Introducción

- Metodología

- Resultados

- Conclusiones

- Referencias bibliográficas

\section{RESUMEN}

Esta investigación está orientada a valorar si, con una adecuada formación, los policías pueden mejorar la identificación de las emociones expresadas facialmente por una persona frente a aquellos participantes que no reciben ningún tipo de formación. Para este estudio hemos incluido 11 emociones ( 7 básicas y 4 nuevas «añadidas») con el fin de identificar si las 4 emociones nuevas «añadidas» se detectan en la misma proporción que las básicas.

Los resultados obtenidos demuestran que una adecuada formación en identificación facial de emociones mejora sustancialmente las habilidades de los policías para leer las expresiones faciales o cualquier otro comportamiento no verbal. No obstante, sería interesante ahondar en la formación global de la comunicación (verbal, paraverbal, facial y corporal) y no únicamente en la facial con el fin de que los policías dispongan de más y mejores conocimientos en comunicación verbo-corporal que sea de aplicación práctica en sus funciones como representantes de la ley.

\section{PALABRAS CLAVE}

Comunicación, policías, emoción, corporal, criminología, no verbal, utilidad.

\begin{abstract}
This research is aimed at assessing if, with appropriate training, police can improve the identification of emotions expressed facially by a person versus those participants who do not receive any training. For this study we have included 11 emotions ( 7 basic and 4 new 'added') in order to identify if the 4 new 'added' emotions are detected in the same proportion as the basic ones.

The results show that adequate training in facial identification of emotions substantially improves the cops' ability to read facial expressions or any other non-verbal behavior. However, it would be interesting to delve into the overall formation of communication (verbal, paraverbal, facial and body) and not only in the facial in order for police to have more and better knowledge in verbal communication that is applicable practice in their functions as representatives of the law.
\end{abstract}

\section{KEYWORDS}

Communication, police, emotion, corporal, criminology, non-verbal, utility.

\section{INTRODUCCIÓN}

Hasta los años sesenta y setenta, reconocidos especialistas en materia de comunicación,

1 Doctorando en Criminología en la Universidad de Cádiz (España). 
expresiones y emociones como Margaret Mead, Gregory Bateson, Edward T. Hall, Ray Birdwhistell, Charles Osgood, entre otros estaban convencidos de que las expresiones y el gesto eran factores aprendidos socialmente y culturalmente variables (Ekman, 2004 y 2005). Sin embargo, un reconocido antropólogo, Charles Darwin, abogaba por la tesis contraria, creía que las emociones eran innatas, heredadas:

Todos están de acuerdo en que las principales acciones expresivas que exhiben los hombres y los animales inferiores son ahora innatas o heredadas, es decir, que no han sido aprendidas por el individuo. Muchas de ellas tienen tan poco que ver con el aprendizaje o la imitación que están por completo fuera del alcance de nuestro control desde los primeros días y a lo largo de toda la vida. (Darwin, 1998, p. 351)

Paul Ekman (2004, pp. 17-34) ahondó en la identificación de las emociones mediante el análisis facial y a través de sus diversos estudios llegó a la conclusión de que existen ciertas emociones universales en diferentes culturas y que se aplican facialmente de forma similar. Llegó a esta conclusión a raíz de los estudios de investigación con personas que pertenecían a culturas tan diferentes como las de Papúa Nueva Guinea, Japón, Estados Unidos, Brasil, Argentina, Indonesia y la antigua Unión Soviética.

La prueba consistía en mostrar la fotografía de varias emociones (por ejemplo, tristeza) a personas de diferentes culturas y comprobaba si todos identificaban la misma emoción o no. Los resultados demostraron que los integrantes de diferentes culturas eran capaces de reconocer de igual forma ciertas emociones. En otros casos, pedía a los miembros de una cultura que mostraran facialmente (expresaran con la cara) una determinada emoción o simulaba una situación para que sientan dicha emoción. Por ejemplo: una situación en la que algo provocaba asco. Esto corroboraba las semejanzas de las expresiones faciales en las distintas culturas. Debido a estos y otros estudios transculturales, llegó a identificar, inicialmente, 6 emociones que en poco tiempo se convirtieron en 7 y que él denominó universales: alegría; arrogancia; asco (repugnancia); ira; sorpresa; temor; tristeza.
Con base en las emociones básicas, reconoció los músculos que se activaban facialmente para expresar una u otra emoción y los clasificó de tal forma que cualquiera pudiera aprender qué músculos se activan al expresar las diferentes emociones universales en nuestros congéneres.

Por esas mismas fechas, Carrol Izard sin conocer las investigaciones de Ekman elaboró un estudio similar con otras culturas para determinar si las expresiones de las emociones podían ser universales. Los resultados que obtuvo fueron similares a los de Ekman y por lo tanto sugerían la universalidad de las emociones (Izard, 1971). Estos estudios fueron las primeras evidencias científicas de la universalidad de las emociones (Ekman, 1972, 1973; Ekman \& Friesen, 1971; Ekman, Sorenson \& Friesen, 1969; Izard, 1971), es decir, se convirtieron en un importante respaldo científico a las tesis de Darwin, avalando el innatismo en determinadas expresiones faciales.

A partir de ese momento, se acepta que existen emociones universales y que estas se expresan facialmente de forma similar en las diferentes culturas por medio de los músculos de la cara que se activan y así poder enseñar cómo reconocerlas.

No obstante, como es un tema controvertido Lisa Feldman Barret no está de acuerdo con las investigaciones de Paul Ekman y en sus investigaciones desde el 2006 hasta el 2010 pone en duda esta universalidad de las emociones. Uno de sus argumentos es que Ekman en su estudio transcultural enseñaba fotos de emociones a la tribu de Nueva Guinea que no había tenido contacto con occidentales, pero daba opciones limitadas con sus nombres (ejemplo: enfado, alegría, tristeza...) y al utilizar los nombres de las emociones, Feldman interpreta que la investigación está viciada.

Asimismo, él realizó otro estudio de similares características en la tribu Himba de Namibia, también aislada del contacto con occidentales, pero en vez de dar opciones para elegir la respuesta, dejó que esta fuera libre, es decir, indicaron a los habitantes de la tribu que apilaran las fotos que creían reflejaban la misma emoción en 6 montones diferentes, sin dar ningún otro tipo de explicación.

Los resultados fueron que en 3 montones los resultados coincidieron con la teoría de Paul 
Ekman, ya que apilaron en el mismo montón a los que sonreían; en otro a los que sentían miedo $\mathrm{y}$ en un tercero a los que no mostraban ninguna emoción, sin embargo, hubo otros 3 montones en los que se mezclaron los que fruncían el ceño enfadados, con los que mostraban asco y con los que fruncían el ceño por tristeza, y eso le llevó a Lisa Feldman (2006) a sugerir que puede que las emociones no sean universales.

Este es un tema interesante de investigación en el que se debe continuar profundizando, pero no demuestra que las emociones no sean universales, ni que Paul Ekman estuviera en un error, puesto que confundir algunos de los gestos faciales de asco con enfado es habitual entre las personas no entrenadas (mucho más si se observan en caras que pertenecen a otras culturas), de igual forma ocurre al confundir el ceño fruncido con otro ceño fruncido por tristeza, no es fácil para los no entrenados, de ahí que en la comunicación habitual con nuestros congéneres algunas veces confundamos la actitud del otro y se den malentendidos, los cuales solucionamos explicando verbalmente cuál era nuestra actitud y que esta se malinterpretó. Que haya habido confusiones en la identificación de algunas emociones no indica que las tesis de Ekman fueran erróneas.

De hecho, las investigaciones de Ekman parecen bien fundamentadas, y una muestra de ello es que posteriormente han sido replicadas y corroboradas por diferentes expertos en la materia, entre los que se puede destacar a David Matsumoto1 ${ }^{2}$, referente actual de los estudios que inició Ekman.

Profundizando en el tema del innatismo de las emociones y atendiendo a la literatura previa en este campo, esta también enuncia que las emociones sentidas se muestran facialmente de la misma forma aun cuando no se hayan podido ver esas expresiones con anterioridad. En favor de dicho innatismo y universalidad de las emociones, se desarrollaron diversos estudios con ciegos de nacimiento (Thompson, 1941; Fulcher, 1942; Eibl-Eibesfeldt, 1970; Galati, Sherer y Ricci-Bitti, 1997) basándose en que si las emociones son universales, estas serían igualmente expresadas por ciegos que por aquellos que no lo son. Los resultados obtenidos en estos estudios avalan dicha tesis,

2 Consulte el siguiente enlace: http://www.davidmatsumoto. com/biography.php sobre todo en lo que a expresiones faciales espontáneas se refiere.

De tal forma que en los estudios con niños sordo-ciegos de nacimiento, estos mostraban facialmente las expresiones de alegría y tristeza sin haberlas visto anteriormente. Y si alguien tiene la tentación de pensar que los niños podían haber identificado esas emociones porque previamente las habían aprendido mediante el tacto, Eibl-Eibesfeldt (Knapp, 1982) señala que los bebés que carecían de brazos (talidomídicos) mostraban las emociones de la misma manera.

Ahondando en la universalidad de las emociones reflejadas en las expresiones faciales, estas se corresponden con la taxonomía de las emociones en diferentes idiomas alrededor del mundo (Romney, Boyd, Moore, Batchelder, \& Brazill, 1996; Romney, Moore, \& Rusch, 1997; Shaver, Murdaya, \& Fraley, 2001; Shaver, Wu, \& Schwartz, 1992). Y también se detectaron similitudes en diferentes culturas, en la respuesta psicológica ante la expresión facial de determinadas emociones, tanto en el sistema nervioso autónomo como en la actividad cerebral. (Davidson, 2003; Ekman, Levenson, \& Friesen, 1983; Levenson, Ekman, \& Friesen, 1990; Levenson, Ekman, Heider, \& Friesen, 1992; Tsai \& Levenson, 1997).

Con base en lo expuesto, saber detectar las emociones básicas de otras personas por su gestualidad facial es un aspecto importante de la comunicación que merece 1 a pe na ser explorado. Por tanto, este trabajo de investigación se ha centrado en identificar las emociones básicas y en valorar la utilidad de esta identificación en los cuerpos policiales. Debido a que las emociones mostradas son un indicio anticipado de la conducta inmediatamente futura, es decir, si se siente rabia, primero se muestra facialmente (y corporalmente), después se actúa con rabia. Aquello permitiría anticipar un mayor número de actitudes, bien sean conflictivas o no, con el fin de afrontarlas con mayores garantías de éxito en su resolución.

En síntesis se pueden identificar emociones básicas valorando la expresión facial, puesto que estas son universales (avalado por los estudios mencionados anteriormente), y se expresan tanto por personas que no han experimentado ningún contacto previo con ellas (niños sordo-ciegos y talidomídicos). 
Por lo tanto, se puede decir que si se aprende a identificar correctamente las emociones que las personas expresan facialmente, es posible identificar una predisposición favorable o desfavorable hacia algo o alguien. Esta información es muy valiosa para solucionar situaciones conflictivas.

Con el fin de mejorar la formación en identificación facial, se ha incrementado el número de emociones, añadiéndose cuatro emociones nuevas «añadidas» que consideramos presentan rasgos faciales significativamente diferentes y que creemos pueden ser discriminadas por las personas: desprecio; dolor; indiferencia; resignación.

Atendiendo la importancia de la identificación facial de las emociones, se ha elaborado una metodología en la que intentamos determinar lo siguiente:

- Los policías con formación básica en identificación facial de emociones captan con mayor frecuencia las emociones faciales en los demás y las identifican mejor con una formación adecuada que sin esta.

- Los policías que son capaces de detectar facialmente las emociones en los demás, son capaces de identificarlas correctamente.

- Los policías catalogan y discriminan las emociones nuevas «añadidas» de forma correcta y reconocen en qué medida con respecto a las básicas.

- Determinar hasta qué punto es útil la formación en la identificación facial de emociones.

Esta investigación se justifica por la aportación que puede generar en la mejora de la capacitación de los profesionales responsables de valorar la conducta de sujetos conflictivos o problemáticos en el transcurso de una intervención policial. La mejora de la capacitación se debe a que dichos profesionales dispondrían de una mayor y mejor información del lenguaje facial que está desplegando el sujeto valorado, y por ende un mayor conocimiento de la actitud que muestra dicho sujeto.

No solo sería de utilidad en esa área, puesto que si fueran capaces de identificar de forma correcta la comunicación facial que efectúa una persona dándole un significado coherente a su predisposición inmediata (favorable o desfavorable), la utilidad de ese conocimiento traspasaría la labor de los policías, y el ámbito de actuación abarcaría desde su uso en todos los cuerpos y fuerzas de seguridad (públicas y privadas) hasta las entrevistas de recursos humanos, pasando por los interrogatorios, o su uso en las valoraciones de testigos/imputados dentro del sistema judicial (jueces, fiscales y equipos psicosociales) e incluso en terapias individuales o grupales, entre otros.

\section{METODOLOGÍA}

Se utilizará un método de investigación experimental y cuantitativo.

A partir de este método, se pretende conocer si los sujetos identifican las emociones mostradas y en qué grado.

\section{Elección de la población}

Se brindó a todos los asistentes la posibilidad de participar en un curso de Formación de Policías Locales de Andalucía (España). Los sujetos que participaron en esta investigación fueron un total de 24 policías que llevaban un curso de Análisis Verbo-Corporal y Detección de Engaño de 20 horas durante una semana (octubre, 2015).

\section{Selección de la muestra}

Se efectuó en dos fases diferenciadas que a su vez pueden considerarse dos niveles: uno genérico y otro más concreto.

- 1. Se seleccionó a toda la población que cumplía los requisitos y se realizó una comunicación para animarlos a que participen en el estudio.

- 2. De toda la población, estudiamos a aquellos que voluntariamente accedieron a participar en el estudio. De los 24 participantes de la población, todos accedieron a colaborar en la investigación, obteniendo resultados válidos de 19 participantes. Los 6 restantes solo realizaron una de las dos pruebas y puesto que no se podía comprobar el grado de mejora o empeoramiento que habían experimentado con la formación impartida, se optó por no tomarlas en cuenta con el fin de no desvirtuar la muestra seleccionada. 
De los 19 sujetos válidos $(80 \%$ de la población), 3 fueron mujeres y 16 hombres con edades comprendidas de los 25 a los 50 años. Todos ellos eran policías locales de diferentes localidades de Andalucía.

\section{Desarrollo}

A los voluntarios que accedieron a participar en el estudio se aplicaron dos pruebas: una al principio de la formación y otra al final.

\section{Primera prueba}

Se informó a los participantes acerca de 33 vídeos sobre emociones realizadas en una fracción de segundo (examen 33A), es decir, veían una cara con una expresión neutra que en una fracción de segundo pasaba a mostrar una emoción. Se indicó que identificaran las emociones mostradas. Para este trabajo, se utilizó un software desarrollado por el GRUPODETECTA. Para responder, contaban con un documento de Excel en el que por cada una de las 33 emociones mostradas tenían 11 elecciones posibles; si al mostrar la emoción número 1, habían identificado alguna emoción, debían elegir una y anotarla en la casilla; si no habían visto ninguna emoción, deberían dejar la casilla en blanco sin responder (anexo 1).

\section{Segunda prueba}

Una vez completada la acción formativa (10 h), se informó a los grupos sobre otros 33 videos de emociones básicas mostradas (examen
33B) en una fracción de segundo. Asimismo, se volvió a utilizar el software desarrollado por el GRUPODETECTA, y se indicó que identificaran las emociones observadas, de igual forma que en la primera prueba. Si habían visto alguna emoción, debían elegir entre una de las 11 opciones; y si no habían conseguido captar la emoción mostrada, debían dejar la casilla en blanco (anexo 2).

Posteriormente, se aplicó otra prueba que consistía en 11 emociones por video a velocidad normal, y se solicitó que las identificaran (anexo 3).

Este estudio integra otra investigación más amplia que consta de tres grupos más de sujetos que han aplicado las mismas pruebas, variando la cantidad de horas de formación, con el objetivo de determinar, en tiempo y metodología, la formación más adecuada para enseñar a detectar emociones facialmente.

\section{RESULTADOS}

En las primeras 33 emociones, los resultados que se obtuvieron fueron los siguientes:

El $100 \%$ de los sujetos identificaron correctamente menos de 26 emociones (de ellos el $45 \%$ no llegaron a identificar bien ni la mitad de las emociones mostradas) como se aprecia en la siguiente gráfica.

\section{Gráfica 1}

Primeras 33 emociones (porcentaje de aciertos/errores)

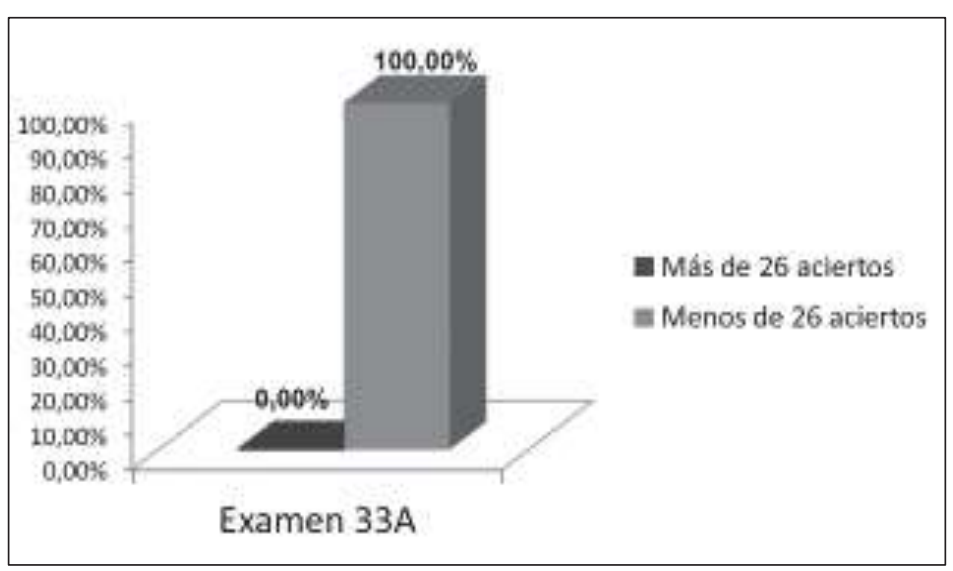

Respecto a la media de aciertos y errores en estas primeras 33 emociones mostradas antes de seguir la formación de identificación de emociones, se puede comprobar cómo ambas medias son muy parecidas. Los sujetos, de media, se equivocaron al identificar emociones lo mismo que acertaron y además la media de aciertos se encuentra en torno al $50 \%$ de emociones, dando lugar a un resultado de aciertos muy bajo. 
Gráfica 2

Primeras 33 emociones (media de aciertos/errores)

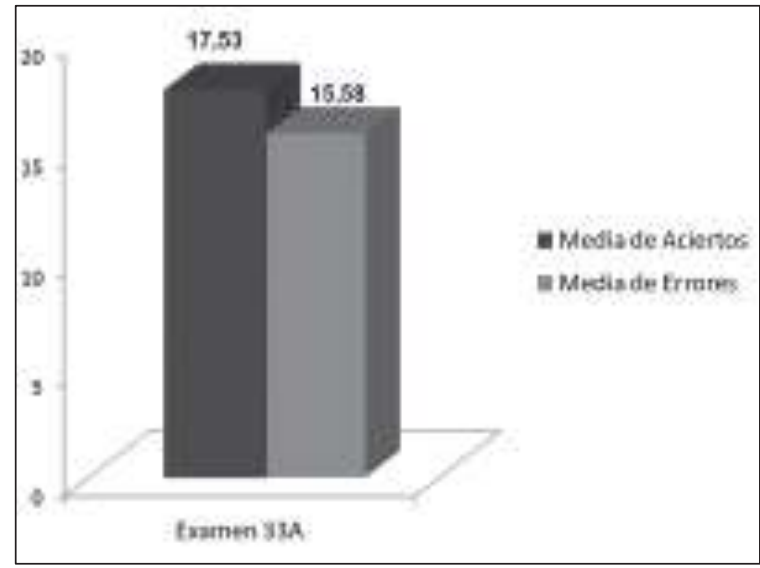

\section{Gráfica 3}

Segundas 33 emociones (porcentaje de aciertos/errores)

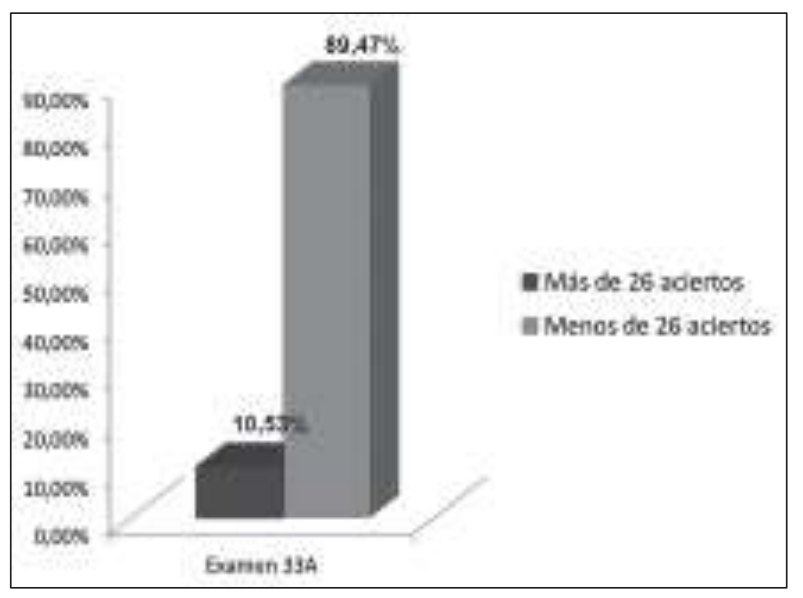

Gráfica 4

Segundas 33 emociones (media de aciertos/errores)

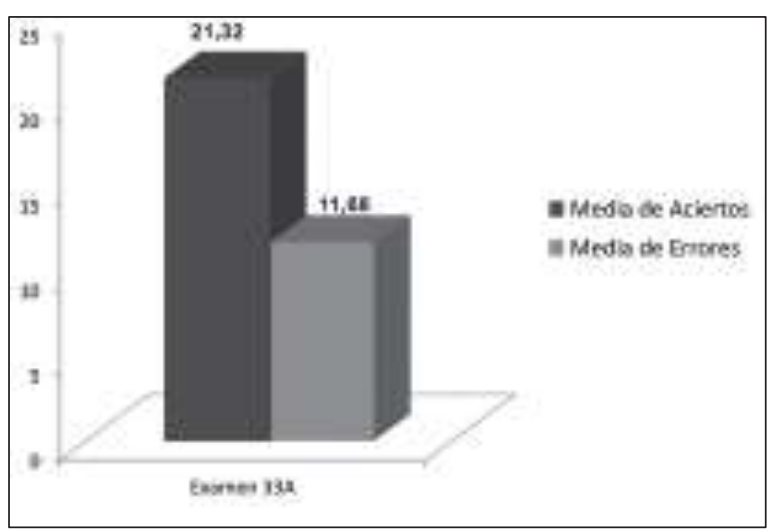

Sin embargo, cuando se plasman sobre una gráfica las emociones detectadas después de haber recibido una formación en identificación de emociones de 10 horas, se pueden detectar algunas mejoras.

En cuanto al porcentaje de sujetos que identificaron más de 26 emociones, lo cual supone identificar correctamente más del $78 \%$ de las emociones mostradas, fue del 10, $5 \%$ (gráfica 3).

La media de identificaciones correctas con respecto a los errores cometidos aumenta significativamente, lo cual indica una mejoría notable en la identificación de emociones. La media de identificaciones correctas se situó en 21,32 emociones, es decir, identificación de más del $63 \%$ de las emociones, mientras que la media de errores en la identificación de emociones fue de 11,68 , como se aprecia en la gráfica 4.

El $79 \%$ de los sujetos mejoraron el porcentaje de identificación de las primeras 33 emociones, un $10 \%$ identificó igual y otro $10 \%$ mejor que antes de la formación (gráfica 5). 


\section{Gráfica 5}

Identificación del $\mathrm{N}^{\circ}$ de emociones

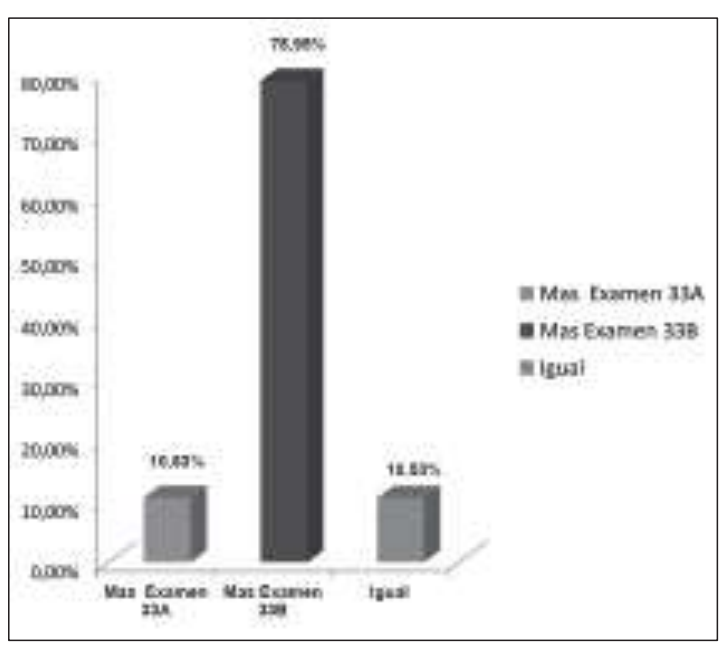

Más del $89 \%$ de los participantes en el estudio identificaron un mayor número de emociones después de 10 horas de formación o el mismo número de emociones. Únicamente un $10 \%$ de los participantes reconocieron mejor las 33 primeras emociones.

En lo referente a las emociones que reconocen una vez que han aprendido cuáles son los rasgos distintivos de cada emoción, los datos muestran que se identificaron correctamente las 7 emociones universales desde las que partía Paul Ekman (Ekman y Friesen, 1975 y Ekman, 2003), en el $100 \%$ de los participantes. Excepto en la tristeza, que un participante la confundió con temor. Además de estas emociones, se añadieron 4 más que se mostraron sin antes haber indicado cuáles eran los rasgos distintivos de cada una y los datos obtenidos demostraron que de las 11 emociones presentadas la media de identificación correcta fue de 10,46 frente a un 0,54 de identificación errónea, es decir, se identificaron correctamente casi todas las emociones, las 11 propuestas.

En la siguiente gráfica, se reflejan los porcentajes de identificación de cada emoción correspondientes a todos los participantes en el estudio. Nótese como las 7 emociones que se explicaron y que se impartieron en el curso fueron identificadas por todos los participantes.
Otro dato reseñable es que de las emociones nuevas «añadidas» (4 emociones más), sin haber hecho hincapié en su formación, algunas de ellas se identificaron con un acierto más que notable siendo la de menor acierto del $75 \%$, mientras que la de mayor acierto fue del 95, 8 $\%$.

\section{Gráfica 6}

Media de emociones identificadas

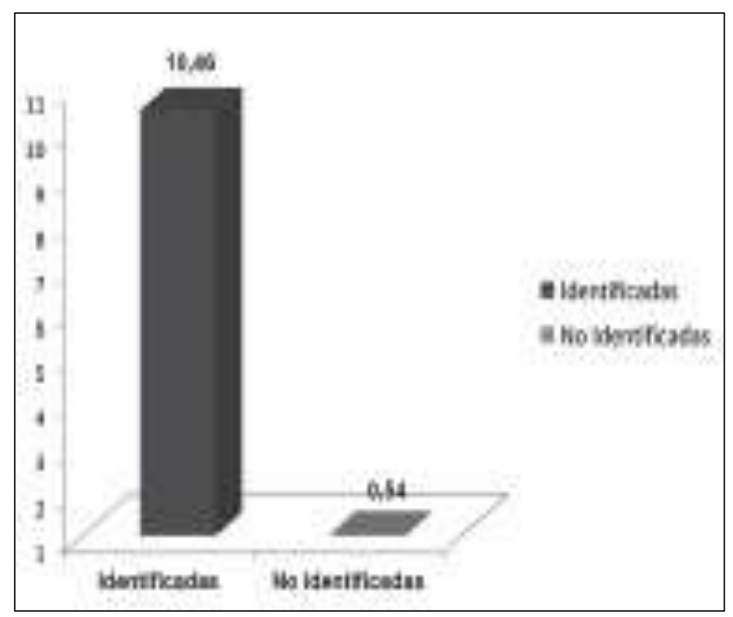

Además, en las emociones que menos se identificaron, se ha comprobado que los participantes confundían casi la misma emoción. Por ejemplo, el desprecio se confundió siempre con arrogancia, entonces se han puesto otras barras al lado (barras rojas), sumándoles esa emoción con otra que la confundía para ver qué porcentaje de identificación correcta se podría haber obtenido si se hubieran incluido estas emociones en la formación y si se hubiera enseñado a los participantes cuáles son los rasgos diferenciales entre las 4 emociones nuevas «añadidas» y las básicas. Por lo tanto, en la siguiente gráfica se presentan por un lado el porcentaje de emociones identificadas (en barras azules), y por otro el porcentaje de emociones identificadas, añadiéndoles esa emoción qu e confundía la mayoría de los participantes (barras rojas). Y se puede comprobar que de las 4 emociones nuevas «añadidas», 2 de ellas se identificarían correctamente por el $100 \%$ de los participantes y las otras 2 por el $96 \%$ de los participantes. 
Gráfica 7

Porcentaje de emociones reconocidas

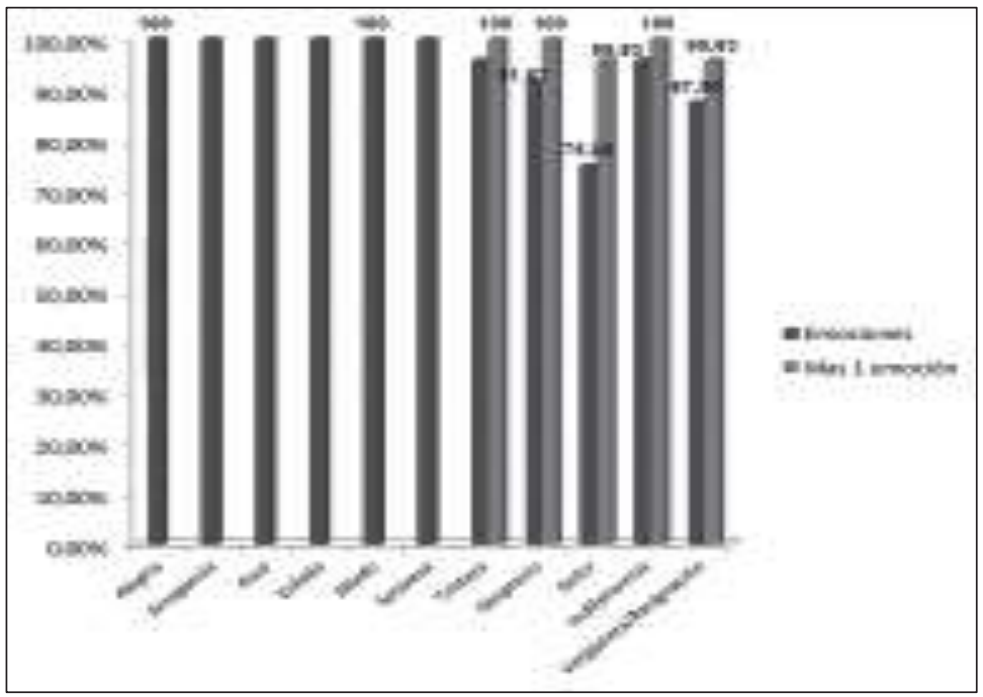

\section{CONCLUSIONES}

Esta investigación demuestra que es posible mejorar la identificación facial de emociones si se dispone del tiempo necesario y se recibe una formación adecuada.

Cuando se carece de formación en identificación facial de emociones, los errores son elevados. En este estudio se obtuvo una media de error en torno al $46 \%$ (casi la mitad) de las emociones que se apreciaron. Si se extrapolara la actuación de los policías en su labor cotidiana, por ejemplo en una entrevista a un sospechoso, se interpretarían mal sus emociones y por consiguiente lo que ese sujeto está sintiendo. De tal forma que si no se reconoce de forma adecuada una emoción, ¿cómo se va a saber reaccionar de forma adecuada? La respuesta es no sería posible. Se reaccionaría ante la emoción identificada, pero como se ha identificado de forma errónea, la posterior actuación de los policías podría empeorar la situación o cuando menos sería una intervención policial incorrecta.

Sin embargo, si se ayuda a que los policías aprendan a identificar mejor las emociones, esto daría un plus de calidad al desarrollo de sus funciones, en su labor cotidiana y en relación con los ciudadanos.

Por supuesto que la identificación de las emociones no es lo único que se debe tener en cuenta cuando se está interviniendo a un ciudadano, sospechoso, víctima o testigo. De hecho, la identificación de las emociones sería solo un apartado para valorar dentro de la comunicación facial. Para una completa valoración de la comunicación, es necesario tener en cuenta toda la comunicación que el sujeto despliega tanto en su componente verbal (y paraverbal) como en el corporal (y facial), e interpretarlo todo dentro de un contexto cultural, como apuntó muy bien Edward T. Hall (1989), pero eso es materia para una investigación más extensa que la que aquí se presenta.

En este estudio se ha valorado la identificación de las emociones faciales básicas, porque está ampliamente reconocido que son universales, y lo que se pretendía era saber si con una formación básica en este tipo de identificación era posible que los policías mejoraran sus habilidades en la identificación de emociones, ya que si era así, obtendrían una mayor y más fiable información de lo que comunica la otra persona y por consiguiente su intervención podría ser más eficiente.

Los resultados arrojan que la mayoría de los participantes mejoraron sustancialmente en su identificación facial de emociones, pasando de identificar de media el $51 \%$ de la emociones, a identificar de media el $64 \%$ de las emociones, y eso teniendo en cuenta 4 emociones nuevas «añadidas» de las que no se dio ningún tipo de formación. 
Con respecto a los fallos cometidos, en las primeras 33 emociones los fallos fueron de 15 emociones erróneas de media, mientras que en las segundas 33 emociones el error descendió hasta 11 , lo que nuevamente refuerza la mejora obtenida en la identificación de emociones.

Por otro lado, los representantes de la policía que participaron en este estudio, no solo identificaron correctamente casi el $100 \%$ de las emociones denominadas como «básicas» por Paul Ekman (1 participante falló en una emoción), sino que además identificaron otras emociones que hemos denominado nuevas «añadidas», en un alto porcentaje del $75 \%$ y el $95,8 \%$.

Se concluye que es posible aumentar significativamente la identificación de emociones faciales mediante el aprendizaje y que se consigue el reconocimiento de prácticamente todas las emociones básicas por parte de los participantes del estudio con una formación de 10 horas.

Que el alto porcentaje de identificación de las cuatro nuevas emociones (denominadas añadidas), sin haber impartido formación específica a los participantes del estudio, apunta en la dirección de que esas emociones presentan rasgos faciales distintivos y específicos de cada una, reconocibles por las personas de forma diferente al resto de las emociones básicas. Es decir, si en futuros estudios con una muestra mayor y con personas de diferentes culturas se corroboraran estos datos, sería factible que esas nuevas emociones también fueran distinguibles por todas las personas y culturas, por lo que podrían llegar a considerarse básicas.

No obstante, se debe continuar ahondando en la identificación de emociones y en la formación de esta habilidad para conseguir mejores resultados, y para intentar determinar qué tiempo y qué formación necesitamos para obtener una identificación del $90 \%$ de las emociones y un reconocimiento del $100 \%$ de las 11 emociones presentadas y así se podría determinar que la identificación de emociones es óptima.

Como última conclusión derivada de una mejora en las capacidades de los policías, se puede aseverar que dicha formación los ayuda poniendo a su disposición herramientas útiles y de uso cotidiano con el objetivo de hacer una intervención policial lo más eficiente posible, la cual consistiría en las siguientes razones:
- Los policías tienen una mayor seguridad en su intervención, porque tienen más elementos de juicio y más creíbles acerca de la comunicación de las personas (identificación facial de emociones).

- La relación ciudadano-policía mejora debido a que los policías pueden interpretar mejor las emociones que detectan en los ciudadanos con que se relacionan y pueden ser más empáticos, favoreciendo la comunicación y la atención al ciudadano.

- Una intervención en situaciones conflictivas o problemáticas ayuda a los policías a identificarlas cuando son incipientes, y a reconducirlas de la forma más adecuada. Por ejemplo: una persona enfadada muestra ira en el rostro antes de verbalizarla y antes de golpear o insultar a alguien, momento en el que el policía puede intervenir para evitar incidentes mayores.

Finalmente, para que la investigación tuviera un mayor peso porcentual y una mayor base científica sería interesante ampliar la muestra de los participantes o la población a grupos policiales de otras regiones de España. Lo ideal es que se pudiera desarrollar, por un lado, con más grupos de los mismos profesionales, $\mathrm{y}$ por otro lado, con profesionales diferentes, contrastando la homogeneidad de los resultados obtenidos.

Este trabajo deja abiertas las puertas para otras investigaciones en el campo de la identificación facial de emociones y su relación con la conducta inmediatamente futura de las personas, las situaciones conflictivas o problemáticas, y la capacitación de los profesionales que tengan que lidiar con dichas situaciones.

\section{REFERENCIAS BIBLIOGRÁFICAS}

Darwin, Ch. (1998). La expresión de las emociones en los animales y en el hombre. Madrid: Alianza Editorial.

Davidson, R. J. (2003). Parsing the subcomponents of emotion and disorders of emotion: Perspectives from affective neuroscience. [In R. J. Davidson, K. R. Scherer, \& H. H. Goldsmith (eds.), Handbook of affective sciences], (8-24). New York: Oxford University Press. 
Eibl-Eibesfeldt, I. (1970). Ethology, the Biology of Behavior. Nueva York: Holt, Rinehart \& Winston.

Ekman, P. (1972). Universals and cultural differences in facial expressions of emotion. [In J. Cole (ed.), Nebraska Symposium on Motivation, 1971]. Lincoln, Neb.: University of Nebraska Press (207-283).

Ekman, P. (1973). Darwin and facial expression; A century of research in review. New York: Academic Press.

Ekman, P. (2004). ¿Qué dice ese gesto?

Barcelona: RBA Libros.

Ekman, P. (2005). Cómo detectar mentiras. Barcelona: Editorial Paidós.

Ekman, P. \& Friesen, W. (1971). Constants across culture in the face and emotion. [In Journal of Personality and Social Psychology], $(17,124-129)$.

Ekman, P. y Friesen, W.V. (1975) Unmasking the Face: A Guide to Recognizing Emotions from Facial Clues. Prentice-Hall.

Ekman, P., Levenson, R. W. \& Friesen, W. V. (1983). Autonomic nervous system activity distinguishes among emotions. [In Science], (221, 1208-1210).

Ekman, P., Sorenson, E. R. \& Friesen, W. V. (1969). Pancultural elements in facial displays of emotion. [In Science], (164, 86-88).

Feldman, L. Are Emotions Natural Kinds? [Perspectives in Psychological Sciences, 2006]. (1,1), 28-58.

Fulcher, J.S. (1942). Voluntary Facial Expression in Blind and Seeing Children. [In Archives of Psychology], vol. 38.

Galati, D., Scherer, K.R. y Ricci-Bitti, P.E. (1997). Voluntary Facial Expression of Emotion: Comparing Congenitally Blind with Normally Sighted Encoders. [In Journal of Personality and Social Psychology], (73, 1363-1379).

Hall, E. T. (1989). Beyond Culture. New York: Anchor Books Editions.

Izard, C. (1971). The Face of Emotion. Nueva York: Appleton-Century-Crofts. [En Ekman, P. (2004) ¿Qué dice ese gesto?] Barcelona: RBA Integral.
Knapp, M. L. (1982). La comunicación no verbal. El cuerpo y el entorno. España: Paidós Ibérica, S.A.

Levenson, R. W., Ekman, P. \& Friesen, W. V. (1990). Voluntary facial action generates emotion-specific autonomic nervous system activity. [In Psychophysiology], (27, 363-384).

Levenson, R. W., Ekman, P., Heider, K. \& Friesen, W. V. (1992). Emotion and autonomic nervous system activity in the Minangkabau of West Sumatra. [In Journal of Personality and Social Psychology], (62, 972-988).

Romney, A. K., Boyd, J. P., Moore, C. C., Batchelder, W. H. \& Brazill, T. J. (1996). Culture as shared cognitive representations. [In Proceedings of the National Academy of Sciences of the United States of America], (93, 4699-4705).

Romney, A. K., Moore, C. C. \& Rusch, C. D. (1997). Cultural universals: Measuring the semantic structure of emotion terms in English and Japanese. [In Proceedings of the National Academy of Sciences of the United States of America], (94, 5489-5494).

Shaver, P., Murdaya, U. \& Fraley, R. C. (2001). The structure of the Indonesian emotion lexicon. [In Asian Journal of Social Psychology], (4, 201-224).

Shaver, P. R., Wu, S. \& Schwartz, J. C. (1992). Cross-cultural similarities and differences in emotion and its representation. [In M. S. Clark (Ed.), Emotion: Review of personality and social psychology] $(13,175-212)$. Thousand Oaks, CA: Sage.

Thompson, J. (1941). Development of facial expressions of emotion in blind and seeing children. [In Archives of Psychology], vol. 37.

Tsai, J. L. \& Levenson, R. W. (1997). Cultural influences of emotional responding: Chinese, American and European American dating couples during interpersonal conflict. [In Journal of Cross-Cultural Psychology], (28, 600-625). Recuperado de http://www. davidmatsumoto.com/biography.php 